Supporting Information

\title{
Efficient Separation of Propene and Propane Using Anion-Pillared Metal-Organic Frameworks
}

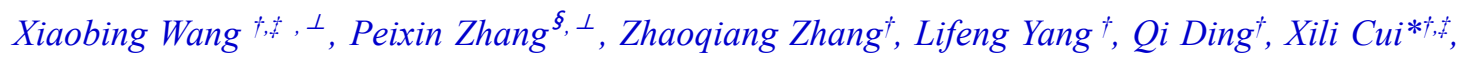
Jun Wang ${ }^{*}$, Huabin Xing ${ }^{\text {t, }}$

$\dagger$ Key Laboratory of Biomass Chemical Engineering of Ministry of Education, College of Chemical and Biological Engineering, Zhejiang University, Hangzhou 310027, China.

‡ Institute of Zhejiang University - Quzhou, 78 Jiuhua Boulevard North, Quzhou 324000, China.

$\S$ School of Resource Environmental and Chemical Engineering, Nanchang University, Nanchang 330031, China.

*Corresponding authors. Email: cuixl@zju.edu.cn; jwang7@ncu.edu.cn. 


\section{Experimental Part}

1. Materials

Ammonium hexafluorogermante $\left(\left(\mathrm{NH}_{4}\right)_{2} \mathrm{GeF}_{6}, 99.99 \%\right.$, Alfa Aesar), Ammonium hexafluorosilicate $\left(\left(\mathrm{NH}_{4}\right)_{2} \mathrm{SiF}_{6}, 98 \%\right.$, Aldrich), Copper tetrafluoroborate hexahydrate $\left(\mathrm{Cu}\left(\mathrm{BF}_{4}\right)_{2} \cdot \mathrm{xH}_{2} \mathrm{O}, \quad 99.99 \%, \quad\right.$ Aldrich $), \quad 4,4$ '-dipyridylacetylene $\quad\left(\mathrm{C}_{12} \mathrm{H}_{8} \mathrm{~N}_{2}, \quad 98 \%\right.$, SUNGYOUNG), and Methanol $\left(\mathrm{CH}_{3} \mathrm{OH}\right.$, anhydrous, 99\%, Sigma-Aldrich) were commercially available of all and used as supplied without further purification.

$\mathrm{N}_{2}$ (99.999\%), Propylene (99.99\%), Propane (99.99\%), He (99.999\%), and mixed gases of Propylene/ Propane $=50 / 50(\mathrm{v} / \mathrm{v})$, were purchased from Shanghai Wetry Standard Reference Gas Analytical Technology Co. LTD (China).

\section{Characterization}

Powder X-ray diffraction (PXRD) was collected through SHIMADZU XRD-6000 diffractometer with $\mathrm{Cu} \mathrm{K \alpha}$ radiation $(\lambda=1.540598 \AA$ ), which operated at the power of $40 \mathrm{kV}, 30 \mathrm{~mA}$ and a scan speed of $4.0 \% \mathrm{~min}$. The data were collected in the range of $2 \theta=5-60^{\circ}$.

3. Water Stability Experiments.

Water stability experiments were carried out by placing $100 \mathrm{mg}$ samples in 20 $\mathrm{mL}$ vials containing $15 \mathrm{~mL}$ of deionized water for $48 \mathrm{~h}$ at ambient temperature, respectively. Subsequently, the samples were obtained through filtration followed by 
activated at $65^{\circ} \mathrm{C}$ for more than $10 \mathrm{~h}$. The water stability was characterized by PXRD and gas adsorption $\left(\mathrm{C}_{3} \mathrm{H}_{6}\right.$ and $\left.\mathrm{C}_{3} \mathrm{H}_{8}\right)$ experiments.

4. Calculations Based on Single Component Isotherms.

4.1 Fitting of Isotherms.

The isotherm data for $\mathrm{C}_{3} \mathrm{H}_{6}$ and $\mathrm{C}_{3} \mathrm{H}_{8}$ on GeFSIX-2-Cu-i and SIFSIX-2-Cu-i were fitted with the Langmuir-Freundlich isotherm model: ${ }^{1}$

$$
\mathrm{q}=q_{A, s a t} \frac{b_{A} p^{v_{A}}}{1+b_{A} p^{v_{A}}}+q_{B, s a t} \frac{b_{B} p^{v_{B}}}{1+b_{B} p^{v_{B}}}
$$

with T-dependent parameters $b_{\mathrm{A}}$, and $\mathrm{b}_{\mathrm{B}}$

$$
b_{A}=b_{A 0} \exp \left(\frac{E_{A}}{R T}\right) ; \quad b_{B}=b_{B 0} \exp \left(\frac{E_{B}}{R T}\right)
$$

Where $\mathrm{q}$ is the adsorbed amount for an adsorbent in $\mathrm{mmol} \mathrm{g}^{-1}, \mathrm{q}_{\mathrm{I}, \text { sat }}$ are the saturated adsorption capacities in $\mathrm{mmol} \mathrm{g}^{-1}, \mathrm{~b}_{\mathrm{I}}$ are the Langmuir parameters in $\mathrm{kPa}^{-1}, \mathrm{p}$ is pressure in $\mathrm{kPa}^{-1}$, and $\mathrm{V}_{\mathrm{I}}$ are the Freundlich parameters for two sites $\mathrm{A}$ and $\mathrm{B}$. Both the pure component isotherms are well fitted by the model and the $\mathrm{R}^{2}$ values are larger than 0.9999 (see Table S3).

\subsection{IAST Calculations of Adsorption Selectivities. ${ }^{2}$}

Ideal adsorbed solution theory model (IAST) calculations of adsorption selectivities for $\mathrm{C}_{3} \mathrm{H}_{6} / \mathrm{C}_{3} \mathrm{H}_{8}$ separation were defined by

$$
S_{a d s}=\frac{q_{1} / q_{2}}{p_{1} / p_{2}}
$$


$\mathrm{q}_{1}$, and $\mathrm{q}_{2}$ are the molar loadings in the adsorbed phase in equilibrium with the bulk gas phase with partial pressures $\mathrm{p}_{1}$ and $\mathrm{p}_{2}$.

\subsection{Isosteric Heat of Adsorption. ${ }^{3}$}

The binding energy of $\mathrm{C}_{3} \mathrm{H}_{6}$ and $\mathrm{C}_{3} \mathrm{H}_{8}$ are reflected indirectly in the isosteric heat of adsorption, Qst, whose calculations are based on the use of the Virial Method, defined as

$$
\ln (\mathrm{p})=\ln (\mathrm{N})+\frac{1}{T} \sum_{i=0}^{m} a_{i} \times N^{i}+\sum_{j=0}^{m} b_{j} \times N^{j}
$$

Where $\mathrm{N}$ represents the adsorption capacity $\left(\mathrm{mg} \mathrm{g}^{-1}\right)$; $\mathrm{p}$ represents pressure $(\mathrm{mmHg})$; $a_{i}, b_{j}$ represent empirical constants, respectively (Table S4).

The isosteric heat of adsorption (Qst):

$$
Q_{s t}=-R \times \sum_{i=0}^{m} a_{i} \times N^{i}
$$

5. Calculations of diffusional time constants. ${ }^{4}$

Diffusional time constants (D', $\left.\mathrm{D} / \mathrm{r}^{2}\right)$ were obtained from the short-time solution of the diffusion equation presuming as step change in the gas-phase concentration, micropore diffusion control and clean beds initially:

$$
\frac{q_{t}}{q_{\infty}}=\frac{6}{\sqrt{\pi}} \cdot \sqrt{\frac{D}{r^{2}} \cdot t}
$$

Where $\mathrm{q}_{\mathrm{t}}$ is the gas uptake at time $\mathrm{t}, \mathrm{q}_{\infty}$ is the gas uptake at equilibrium, $\mathrm{D}$ is the diffusivity and $\mathrm{r}$ is the radius of the equivalent spherical particle. Therefore, the slopes 
of $\mathrm{q}_{\mathrm{t}} / \mathrm{q}_{\infty}$ versus $\mathrm{t}^{1 / 2}$ are derived from the fitting of the plots in the low gas uptake range, and then D' can be obtained from the square of the slope multiplying by $\pi / 36$.
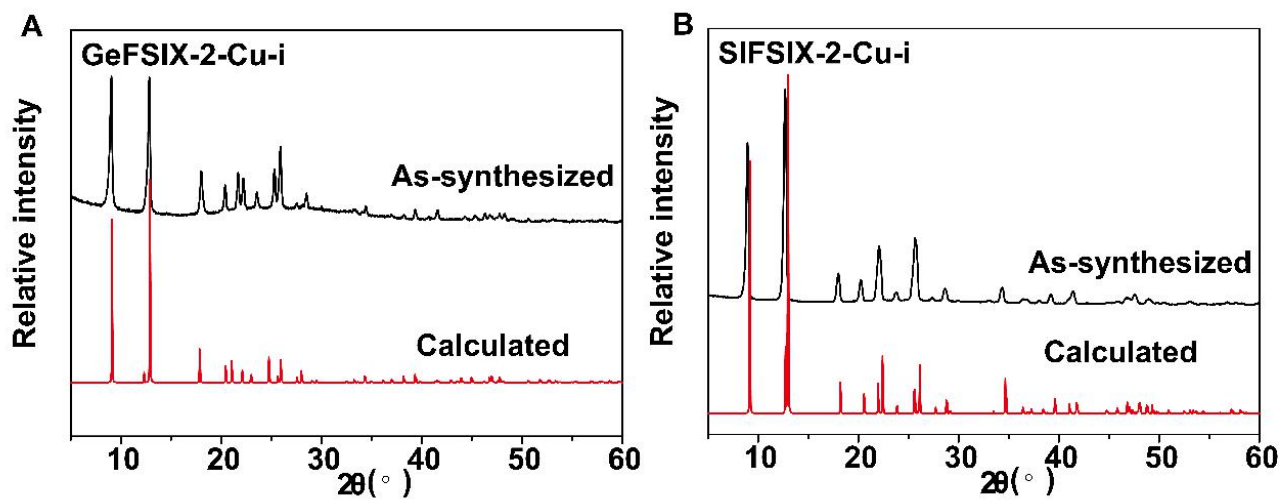

Figure S1. Powder X-ray diffraction patterns of GeFSIX-2-Cu-i and SIFSIX-2-Cu-i.
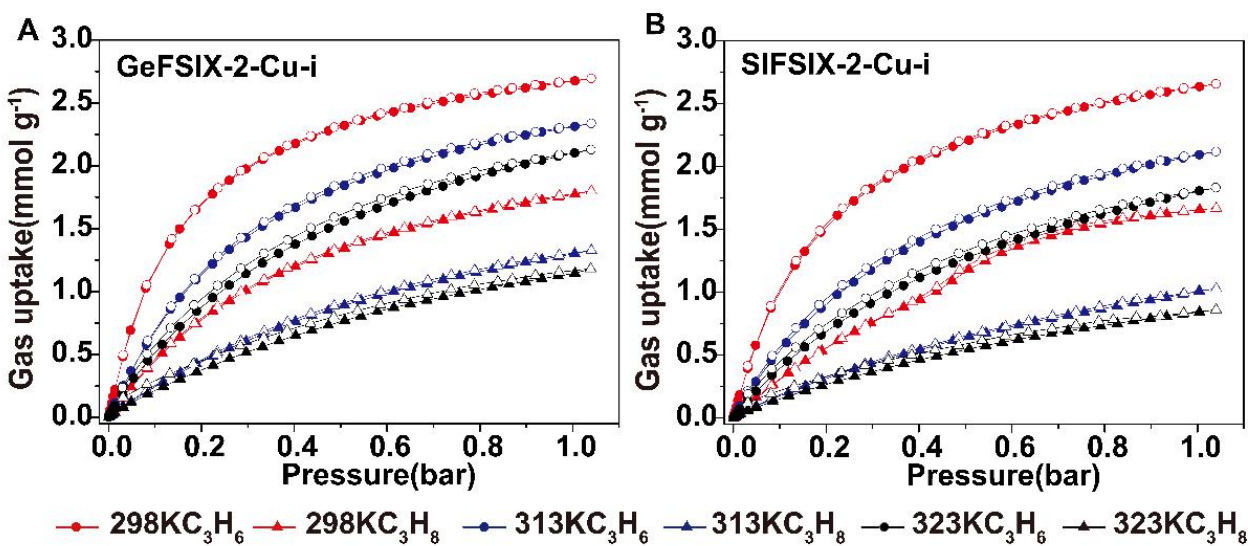

Figure S2. Adsorption isotherms of $\mathrm{C}_{3} \mathrm{H}_{6}$ and $\mathrm{C}_{3} \mathrm{H}_{8}$ on GeFSIX-2-Cu-i (A) and SIFSIX-2-Cu-i (B) at 1.01 bar and different temperatures. 

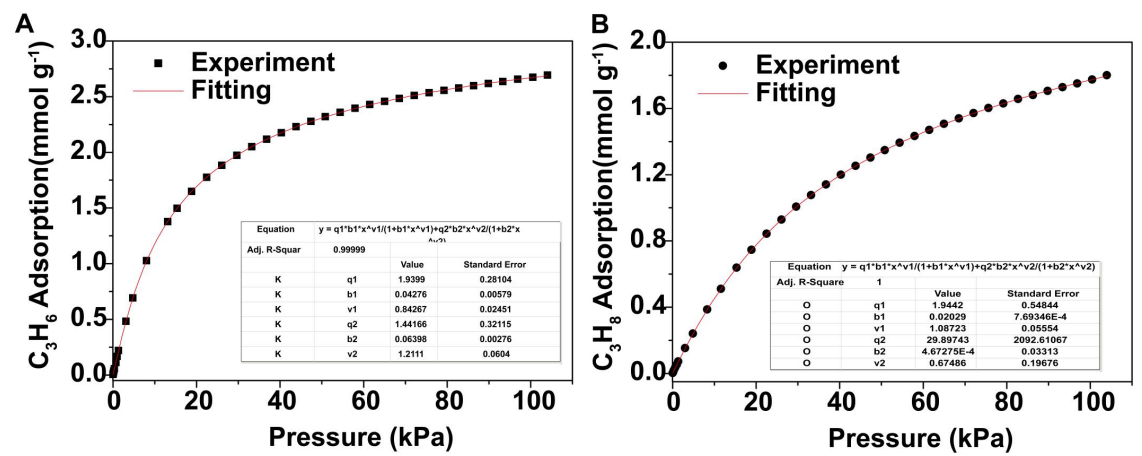

Figure S3. Adsorption isotherms of $\mathrm{C}_{3} \mathrm{H}_{6}$ and $\mathrm{C}_{3} \mathrm{H}_{8}$ on GeFSIX-2-Cu-i fitted with the Langmuir-Freundlich isotherm model.
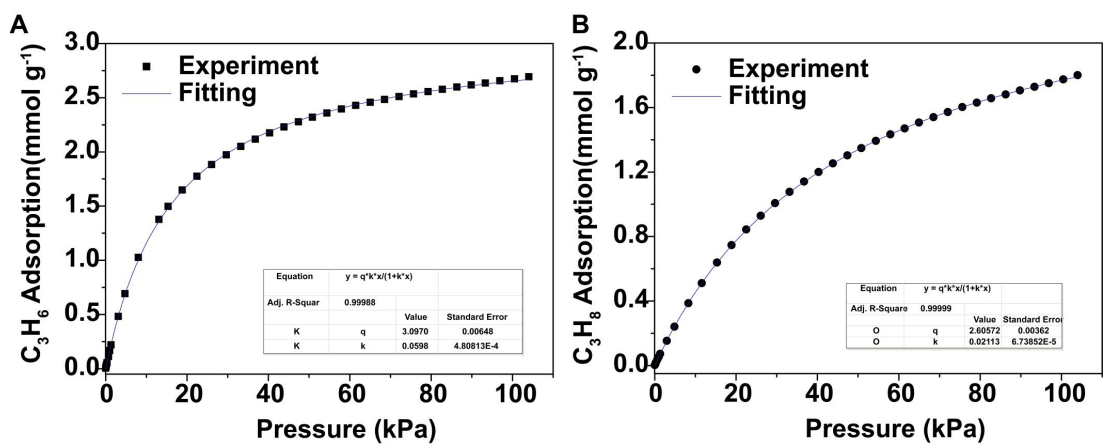

Figure S4. Adsorption isotherms of $\mathrm{C}_{3} \mathrm{H}_{6}$ and $\mathrm{C}_{3} \mathrm{H}_{8}$ on SIFSIX-2-Cu-i fitted with the Langmuir-Freundlich isotherm model.

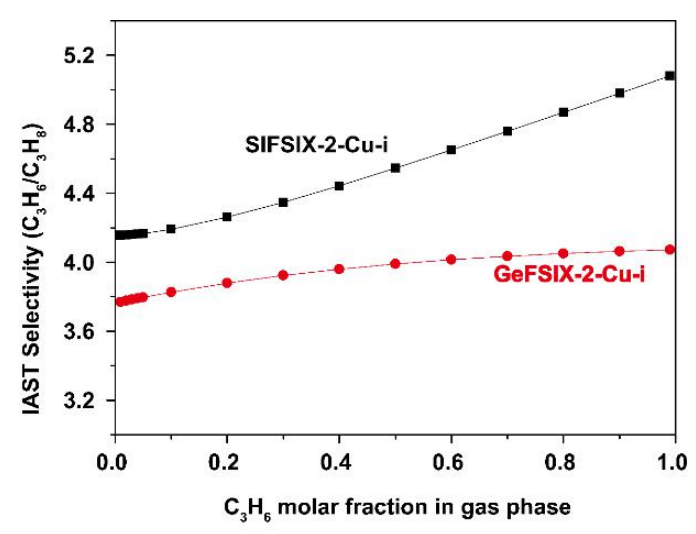

Figure S5. IAST curves at $298 \mathrm{~K}$ and $1.01 \mathrm{bar}$ on GeFSIX-2-Cu-i and SIFSIX-2-Cu-i as a function of the molar fraction of $\mathrm{C}_{3} \mathrm{H}_{6}$. 

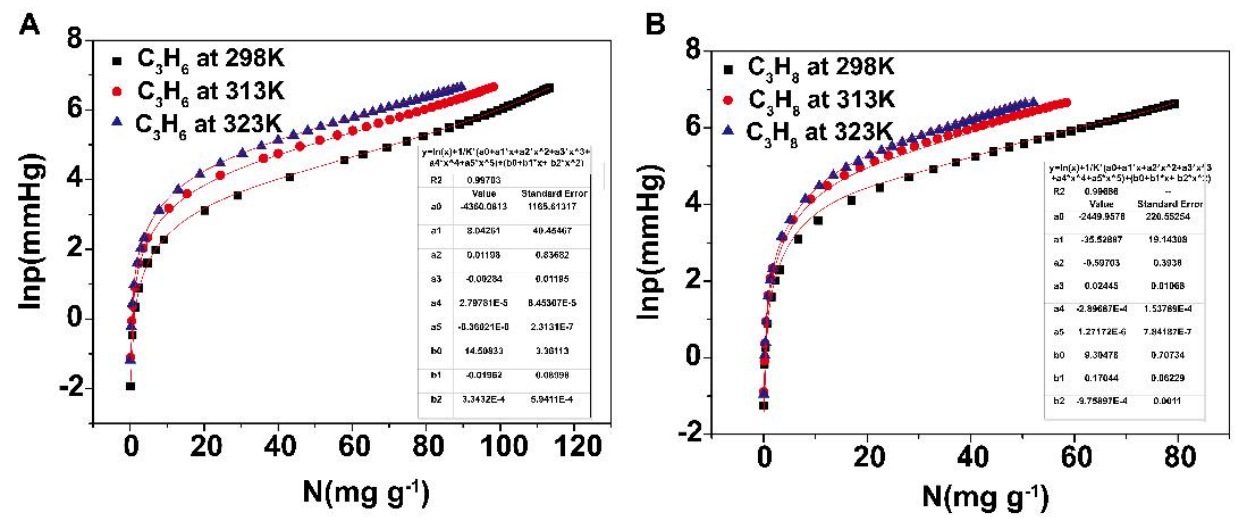

Figure S6. The virial fitting of the $\mathrm{C}_{3} \mathrm{H}_{6}$ and $\mathrm{C}_{3} \mathrm{H}_{8}$ adsorption isotherms for GeFSIX-2-Cu-i at $298 \mathrm{~K}, 313 \mathrm{~K}$ and $323 \mathrm{~K}$.
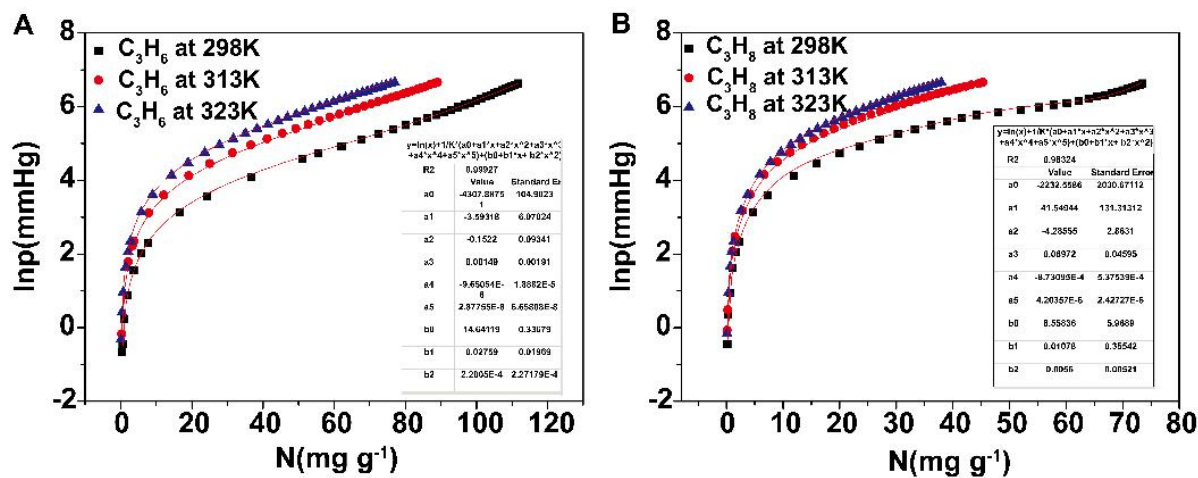

Figure S7. The virial fitting of the $\mathrm{C}_{3} \mathrm{H}_{6}$ and $\mathrm{C}_{3} \mathrm{H}_{8}$ adsorption isotherms for SIFSIX-2-Cu-i at $298 \mathrm{~K}, 313 \mathrm{~K}$ and $323 \mathrm{~K}$.

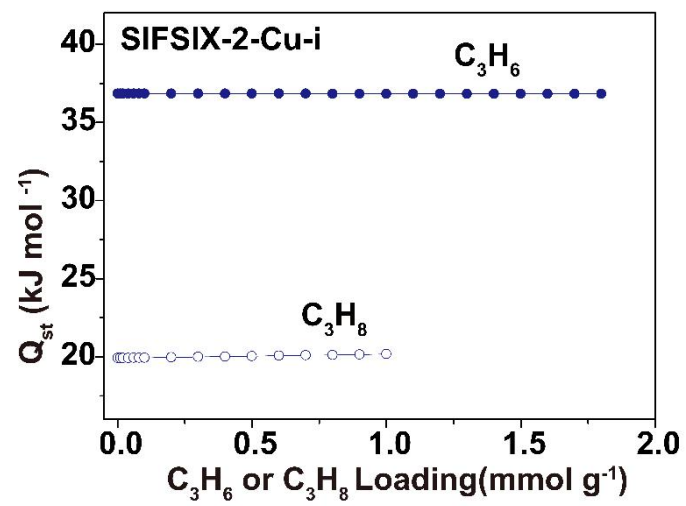

Figure S8. The isosteric heats of adsorption (Qst) of $\mathrm{C}_{3} \mathrm{H}_{6}$ or $\mathrm{C}_{3} \mathrm{H}_{8}$ at low coverage in SIFSIX-2-Cu-i. 

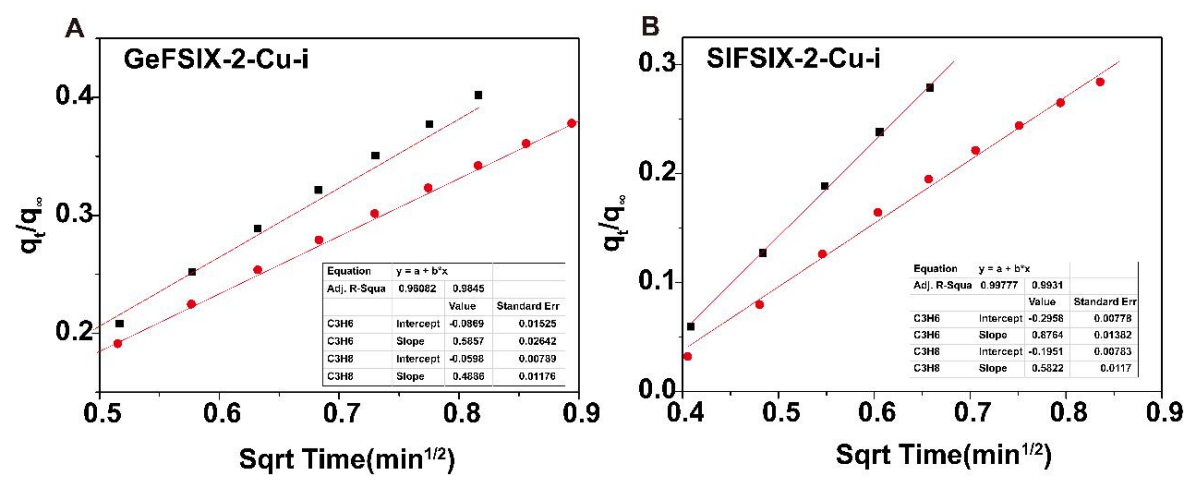

Figure S9. Diffusional time constant calculation details for GeFSIX-2-Cu-i (A) and SIFSIX-2-Cu-i (B). Square and circle symbols represent $\mathrm{C}_{3} \mathrm{H}_{6}$ and $\mathrm{C}_{3} \mathrm{H}_{8}$, respectively.
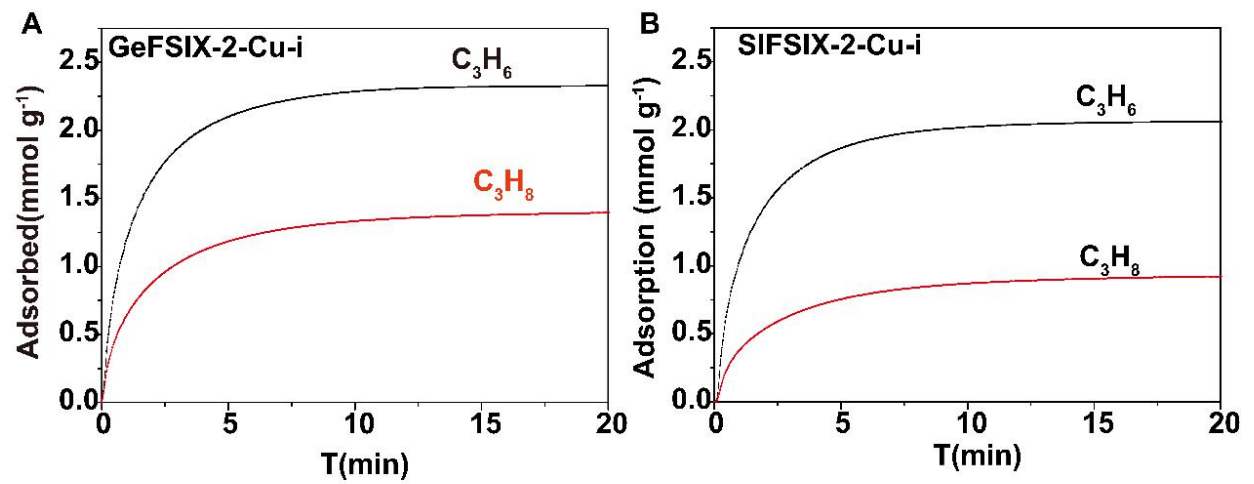

Figure S10. Adsorption kinetics profiles of $\mathrm{C}_{3} \mathrm{H}_{6}$ and $\mathrm{C}_{3} \mathrm{H}_{8}$ for GeFSIX-2-Cu-i (A) and SIFSIX-2-Cu-i (B) at $298 \mathrm{~K}$ and 0.4 bar.
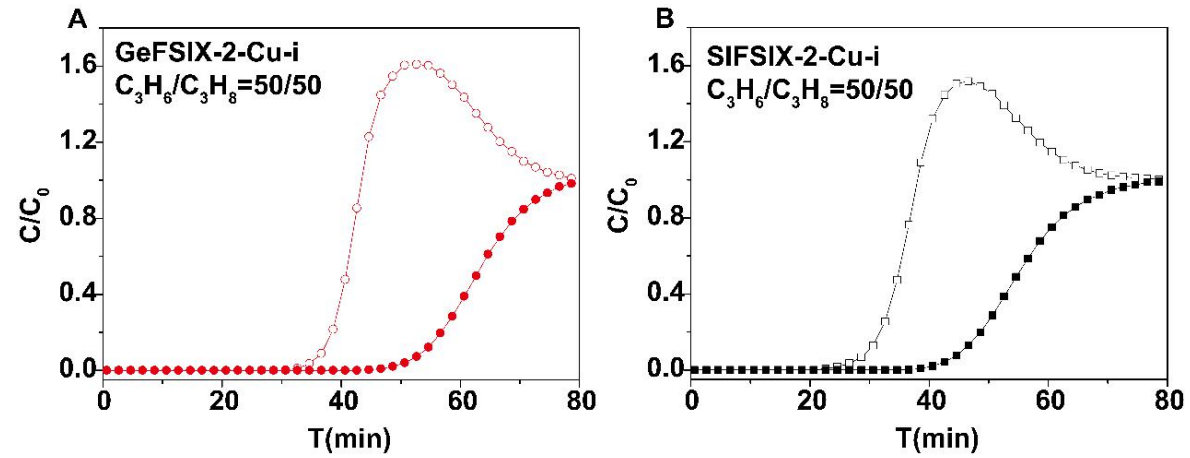

Figure S11. Experimental column breakthrough curves for the mixture of $\mathrm{C}_{3} \mathrm{H}_{6}$ and $\mathrm{C}_{3} \mathrm{H}_{8}(50 / 50, \mathrm{v} / \mathrm{v})$ with GeFSIX-2-Cu-i (A) and SIFSIX-2-Cu-i (B) at $298 \mathrm{~K}$ and $1.01 \mathrm{bar}$. 


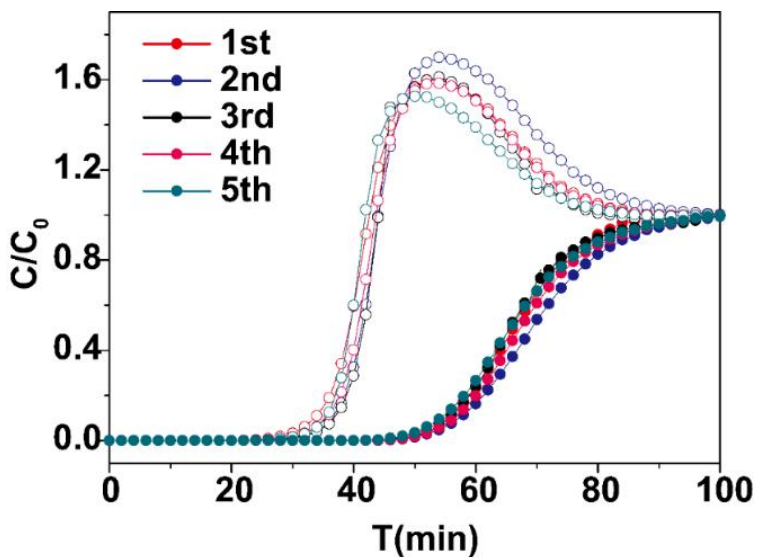

Figure S12. Cycling breakthrough tests for $\mathrm{C}_{3} \mathrm{H}_{6}$ and $\mathrm{C}_{3} \mathrm{H}_{8}(50 / 50$, v/v) separation with GeFSIX-2-Cu-i (mixed gas flow:0.5 $\mathrm{mLmin}^{-1}$ ).

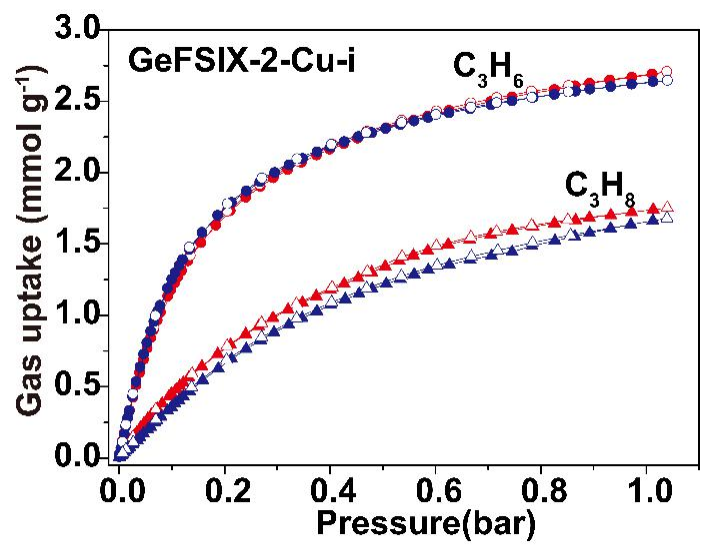

Figure S13. Adsorption isotherms of GeFSIX-2-Cu-i after breakthrough tests collected at 298K (Red and blue curves present the adsorption of before and after breakthrough tests, respectively). 

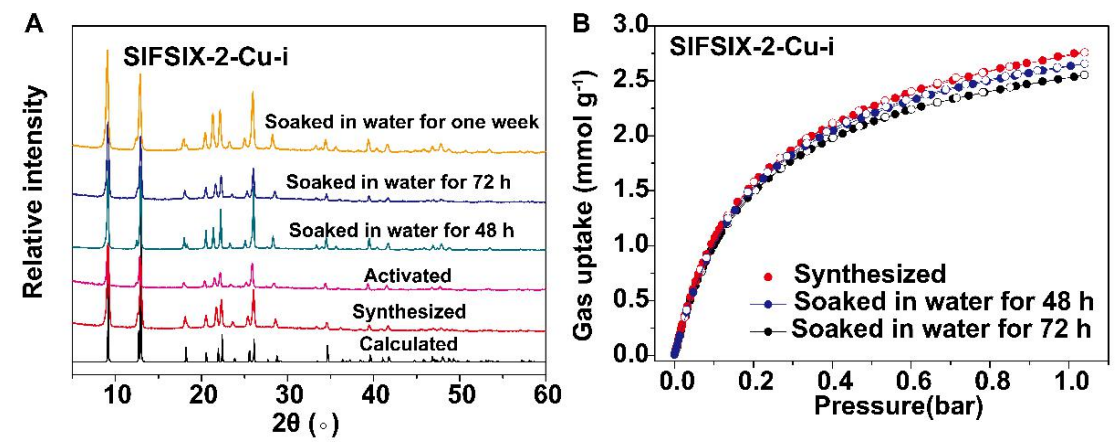

Figure S14. Powder X-ray diffraction patterns (A) and $\mathrm{C}_{3} \mathrm{H}_{6}$ adsorption isotherms (B) of SIFSIX-2-Cu-i after soaked in water collected at $298 \mathrm{~K}$.

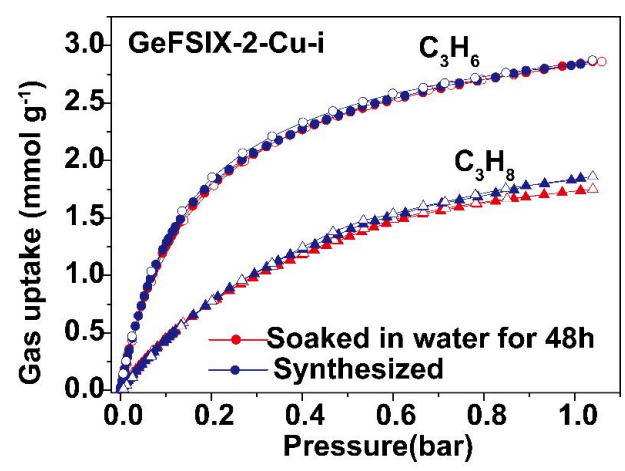

Figure S15. Adsorption isotherms of GeFSIX-2-Cu-i after soaked in water for $48 \mathrm{~h}$ collected at $298 \mathrm{~K}$.

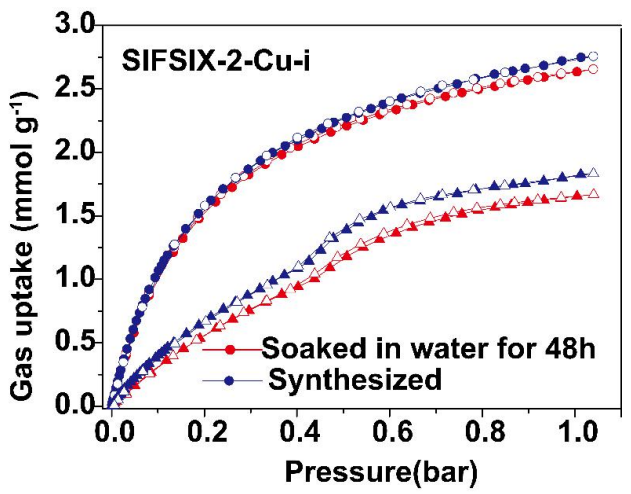

Figure S16. Adsorption isotherms of SIFSIX-2-Cu-i after soaked in water for $48 \mathrm{~h}$ collected at $298 \mathrm{~K}$. 
Table S1. The properties of GeFSIX-2-Cu-i and SIFSIX-2-CU-i.

\begin{tabular}{cccccc}
\hline MOFs & $\begin{array}{c}\text { aperture } \\
(\AA)\end{array}$ & $\begin{array}{c}\text { bond length } \\
(\AA)^{* *}\end{array}$ & $\begin{array}{c}\text { BET } \\
\left(\mathrm{m}^{2} \mathrm{~g}^{-1}\right)\end{array}$ & $\begin{array}{c}\text { pore volume } \\
\left(\mathrm{cm}^{3} \mathrm{~g}^{-1}\right)\end{array}$ & $\begin{array}{c}\text { decomposition } \\
\text { temperature } \\
(\mathrm{K})\end{array}$ \\
\hline GeFSIX-2-Cu-i & 4.5 & 1.78 & 467.6 & 0.25 & 483 \\
SIFSIX-2-Cu-i & 4.7 & 1.75 & $503^{*}$ & 0.26 & 443 \\
\hline
\end{tabular}

**The bond length represents Ge-F and Si-F, respectively.

*Measured by $\mathrm{N}_{2}$.

Table S2. Comparisons with other adsorbents for the separation of $\mathrm{C}_{3} \mathrm{H}_{6} / \mathrm{C}_{3} \mathrm{H}_{8}$ based on thermodynamics

\begin{tabular}{|c|c|c|c|c|c|c|}
\hline \multirow{2}{*}{ Adsorbent } & \multirow{2}{*}{$\begin{array}{l}\text { Temperature } \\
\text { (K) }\end{array}$} & \multirow{2}{*}{$\begin{array}{c}\text { IAST } \\
\text { Selectivity }\end{array}$} & \multicolumn{2}{|c|}{$q\left(\mathrm{mmol} \mathrm{g}^{-1}\right)$ at $1 \mathrm{bar}$} & \multirow{2}{*}{$\begin{array}{l}q\left(\mathrm{C}_{3} \mathrm{H}_{6}\right) \\
-/ \mathrm{q}\left(\mathrm{C}_{3} \mathrm{H}_{8}\right)\end{array}$} & \multirow{2}{*}{ Ref } \\
\hline & & & $\mathrm{C}_{3} \mathrm{H}_{8}$ & $\mathrm{C}_{3} \mathrm{H}_{6}$ & & \\
\hline $\mathrm{Zn}_{2}(5 \text {-aip })_{2}($ bpy $)$ & 298 & 19.8 & 0.76 & 1.91 & 2.51 & [5] \\
\hline ITQ-12 & 303 & 15 & 0.75 & 1.30 & 1.73 & [6] \\
\hline CPL-1 & 298 & 1 & 0.20 & 0.32 & 1.60 & [7] \\
\hline Cu(0.6)@MIL-100(Fe) & 303 & 11.7 & 3.37 & 4.52 & 1.34 & [8] \\
\hline $\mathrm{Mn}_{2}(\mathrm{dobdc})$ & 318 & 16.6 & 5.40 & 7.09 & 1.31 & [9] \\
\hline $\mathrm{Mg}_{2}$ (dobdc) & 318 & 5.5 & 6.00 & 7.40 & 1.23 & [9] \\
\hline $\mathrm{Zn}_{2}(\mathrm{dobdc})$ & 318 & 3.9 & 5.40 & 6.40 & 1.19 & [9] \\
\hline MAF-23-O & 298 & 8 & 1.15 & 1.35 & 1.17 & [10] \\
\hline $\mathrm{Co}_{2}(\mathrm{dobdc})$ & 318 & 8.6 & 5.90 & 6.90 & 1.17 & [9] \\
\hline $\mathrm{Fe}_{2}(\mathrm{dobdc})$ & 318 & 14.7 & 6.10 & 7.10 & 1.16 & {$[9,11]$} \\
\hline MIL-101(Cr)-DAA & 303 & 2.2 & 6.55 & 7.60 & 1.16 & [12] \\
\hline Zeolite 13X & 323 & I & 3.03 & 3.44 & 1.14 & [13] \\
\hline CUBTC & 303 & 3.3 & 8 & 9 & 1.12 & [14] \\
\hline NJUBai-8 & 298 & 4 & 2.89 & 2.89 & 1 & [15] \\
\hline SIFSIX-2-Cu-i & 298 & 4.5 & 1.67 & 2.65 & 1.59 & This \\
\hline GeFSIX-2-Cu-i & 298 & 4.0 & 1.80 & 2.69 & 1.49 & work \\
\hline
\end{tabular}


Table S3. Dual-site Langmuir-Freundlich parameters fitting for $\mathrm{C}_{3} \mathrm{H}_{6}$ and $\mathrm{C}_{3} \mathrm{H}_{8}$.

\begin{tabular}{|c|c|c|c|c|c|c|c|c|}
\hline & & \multicolumn{3}{|c|}{ Site A } & \multicolumn{3}{|c|}{ Site B } & \multirow{3}{*}{$\mathrm{R}^{2}$} \\
\hline & & $q_{A, \text { sat }}$ & $\mathrm{b}_{\mathrm{A} 0}$ & $\mathrm{v}_{\mathrm{A}}$ & $q_{\mathrm{B}, \text { sat }}$ & $b_{\mathrm{B} 0}$ & $\mathrm{v}_{\mathrm{B}}$ & \\
\hline & & $\mathrm{mmol} \mathrm{g}^{-1}$ & $\mathrm{kPa}^{-1}$ & & $\mathrm{mmol} \mathrm{g}^{-1}$ & $\mathrm{kPa}^{-1}$ & & \\
\hline \multirow[t]{2}{*}{ SIFSIX-2-Cu-i } & $\mathrm{C}_{3} \mathrm{H}_{6}$ & 1.994 & 0.029 & 0.828 & 1.641 & 0.050 & 1.152 & 1 \\
\hline & $\mathrm{C}_{3} \mathrm{H}_{8}$ & 1.831 & 0.017 & 1.082 & 0.346 & 3.07E-12 & 6.743 & 0.9999 \\
\hline \multirow[t]{2}{*}{ GeFSIX-2-Cu-i } & $\mathrm{C}_{3} \mathrm{H}_{6}$ & 1.442 & 0.064 & 1.211 & 1.939 & 0.043 & 0.842 & 0.9999 \\
\hline & $\mathrm{C}_{3} \mathrm{H}_{8}$ & 1.944 & 0.020 & 1.087 & 29.897 & 4.67E-4 & 0.675 & 1 \\
\hline
\end{tabular}

Table S4. Fitting parameters of virial equation and the corresponding correlation coefficients of GeFSIX-2-Cu-i and SIFSIX-2-Cu-i.

\begin{tabular}{ccccc}
\hline \multirow{2}{*}{ Parameters } & \multicolumn{2}{c}{ GeFSIX-2-Cu-i } & \multicolumn{2}{c}{ SIFSIX-2-Cu-i } \\
\cline { 2 - 5 } & $\mathrm{C}_{3} \mathrm{H}_{6}$ & $\mathrm{C}_{3} \mathrm{H}_{8}$ & $\mathrm{C}_{3} \mathrm{H}_{6}$ & $\mathrm{C}_{3} \mathrm{H}_{8}$ \\
\hline $\mathrm{a}_{0}$ & -4360.0813 & -2449.9576 & -4307.88751 & -2232.5586 \\
$\mathrm{a}_{1}$ & 8.04261 & -35.52887 & -3.59318 & 41.54944 \\
$\mathrm{a}_{2}$ & 0.01198 & -0.59703 & -0.1522 & -4.28555 \\
$\mathrm{a}_{3}$ & -0.00284 & 0.02445 & 0.00149 & 0.06972 \\
$\mathrm{a}_{4}$ & $2.80 \mathrm{E}-05$ & $-2.90 \mathrm{E}-04$ & $-9.65 \mathrm{E}-06$ & $-8.73 \mathrm{E}-04$ \\
$\mathrm{a}_{5}$ & $-8.36 \mathrm{E}-08$ & $1.27 \mathrm{E}-06$ & $2.88 \mathrm{E}-08$ & $4.20 \mathrm{E}-06$ \\
$\mathrm{~b}_{0}$ & 14.50833 & 9.30478 & 14.64119 & 8.55836 \\
$\mathrm{~b}_{1}$ & -0.01962 & 0.17044 & 0.02759 & 0.01078 \\
$\mathrm{~b}_{2}$ & $3.34 \mathrm{E}-04$ & $-9.76 \mathrm{E}-04$ & $2.20 \mathrm{E}-04$ & 0.0056 \\
$\mathrm{R}^{2}$ & 0.99703 & 0.99686 & 0.99927 & 0.9987 \\
\hline
\end{tabular}

Table S5. The results of diffusional time constant calculations of GeFSIX-2-Cu-i and

SIFSIX-2-Cu-i.

\begin{tabular}{cccccccc}
\hline & \multicolumn{5}{c}{$\mathrm{C}_{3} \mathrm{H}_{6}$} & \multicolumn{3}{c}{$\mathrm{C}_{3} \mathrm{H}_{8}$} & \\
\cline { 2 - 6 } & Slope & $\mathrm{R}^{2}$ & $\begin{array}{c}\mathrm{D}^{\prime} \times 10^{-2} \\
\left(\mathrm{~min}^{-1}\right)\end{array}$ & Slope & $\mathrm{R}^{2}$ & $\begin{array}{c}\mathrm{D}^{\prime} \times 10^{-2} \\
\left(\mathrm{~min}^{-1}\right)\end{array}$ & $\mathrm{D}_{\mathrm{C} 3 \mathrm{H} 6}^{\prime} / \mathrm{D}_{\mathrm{C} 3 \mathrm{H} 8}$ \\
\hline GeFSIX-2-Cu-i & 0.5857 & 0.9608 & 2.99 & 0.4886 & 0.9845 & 2.08 & 1.44 \\
SIFSIX-2-Cu-i & 0.8764 & 0.9978 & 6.70 & 0.5822 & 0.9931 & 2.96 & 2.26 \\
\hline
\end{tabular}


Table S6. The comparisons of diffusional time constants and kinetic selectivity of $\mathrm{C}_{3} \mathrm{H}_{6} / \mathrm{C}_{3} \mathrm{H}_{8}$ at selected temperatures.

\begin{tabular}{|c|c|c|c|c|c|}
\hline \multirow{2}{*}{ Adsorbent } & \multicolumn{2}{|c|}{$D^{\prime} \times 10^{-4}\left(\mathrm{~min}^{-1}\right)$} & \multirow{2}{*}{$\begin{array}{c}\text { kinetic } \\
\text { selectivity }\end{array}$} & \multirow{2}{*}{$\mathrm{T}(\mathrm{K})$} & \multirow{2}{*}{ Ref } \\
\hline & $\mathrm{C}_{3} \mathrm{H}_{6}$ & $\mathrm{C}_{3} \mathrm{H}_{8}$ & & & \\
\hline BTO & 1.3 & 0.11 & 12 & 298 & [16] \\
\hline DBTO & 3.3 & 0.29 & 11 & 298 & [16] \\
\hline $\mathrm{Zn}(\mathrm{ox})_{0.5}(\mathrm{atrz})$ & 8.93 & 0.07 & 126 & 303 & [17] \\
\hline TO & 42 & 17 & 2.47 & 298 & [16] \\
\hline DTO & 110 & 81 & 1.36 & 298 & [16] \\
\hline $\mathrm{Zn}(\mathrm{ox})_{0.5}(\operatorname{trz})$ & 136 & 0.16 & 860 & 303 & [17] \\
\hline ELM-12 & 662 & 3.24 & 204 & 298 & [18] \\
\hline MAF-23-O & 1229 & 17 & 71 & 298 & [10] \\
\hline $\mathrm{Co}(\mathrm{AIP})(\mathrm{BPY})_{0.5}$ & 1814 & 61 & 29.7 & 303 & [19] \\
\hline SIFSIX-2-Cu-i & 670 & 296 & 2.26 & 298 & \multirow{2}{*}{ This work } \\
\hline GeFSIX-2-Cu-i & 299 & 208 & 1.44 & 298 & \\
\hline
\end{tabular}


Reference:

1. Cui, X.; Yang, Q.; Yang, L.; Krishna, R.; Zhang, Z.; Bao, Z.; Wu, H.; Ren, Q.;

Zhou, W.; Chen, B.; Xing, H., Ultrahigh and Selective $\mathrm{SO}_{2}$ Uptake in Inorganic

Anion-Pillared Hybrid Porous Materials. Adv. Mater. 2017, 29 (28), 1606929.

2. Walton, K. S.; Sholl, D. S., Predicting multicomponent adsorption: 50 years of the ideal adsorbed solution theory. AIChE J. 2015, 61 (9), 2757-2762.

3. Rowsell, J. L. C.; Yaghi, O. M., Effects of Functionalization, Catenation, and Variation of the Metal Oxide and Organic Linking Units on the Low-Pressure Hydrogen Adsorption Properties of Metal-Organic Frameworks. J. Am. Chem. Soc. 2006, 128, 1304-1315.

4. Lee, C. Y.; Bae, Y. S.; Jeong, N. C.; Farha, O. K.; Sarjeant, A. A.; Stern, C. L.; Nickias, P.; Snurr, R. Q.; Hupp, J. T.; Nguyen, S. T., Kinetic separation of propene and propane in metal-organic frameworks: controlling diffusion rates in plate-shaped crystals via tuning of pore apertures and crystallite aspect ratios. J. Am. Chem. Soc. 2011, 133 (14), 5228-5231.

5. Chen, Y.; Wu, H.; Lv, D.; Yuan, N.; Xia, Q.; Li, Z., A pillar-layer metal-organic framework for efficient adsorption separation of propylene over propane. Sep. Purif. Technol. 2018, 204, 75-80.

6. Gutie'rrez-Sevillano., J. J.; Dubbeldam., D.; Rey., F.; Valencia., S.; Palomino., M.; 'n-Calvo., A. M.; Calero, S. a., Analysis of the ITQ-12 Zeolite Performance in Propane-Propylene Separations Using a Combination of Experiments and Molecular Simulations. The Journal of Physical Chemistry C. 2010, 114, 14907-14914. 
7. Chen, Y.; Qiao, Z.; Lv, D.; Duan, C.; Sun, X.; Wu, H.; Shi, R.; Xia, Q.; Li, Z., Efficient adsorptive separation of $\mathrm{C}_{3} \mathrm{H}_{6}$ over $\mathrm{C}_{3} \mathrm{H}_{8}$ on flexible and thermoresponsive CPL-1. Chem. Eng. J. 2017, 328, 360-367.

8. Kim, A.-R.; Yoon, T.-U.; Kim, E.-J.; Yoon, J. W.; Kim, S.-Y.; Yoon, J. W.; Hwang, Y. K.; Chang, J.-S.; Bae, Y.-S., Facile loading of $\mathrm{Cu}(\mathrm{I})$ in MIL-100(Fe) through redox-active $\mathrm{Fe}(\mathrm{II})$ sites and remarkable propylene/propane separation performance. Chem. Eng. J. 2018, 331, 777-784.

9. Geier, S. J.; Mason, J. A.; Bloch, E. D.; Queen, W. L.; Hudson, M. R.; Brown, C. M.; Long, J. R., Selective adsorption of ethylene over ethane and propylene over propane in the metal-organic frameworks $\mathrm{M}_{2}(\mathrm{dobdc})(\mathrm{M}=\mathrm{Mg}, \mathrm{Mn}$, Fe, Co, Ni, Zn). Chemical Science. 2013, 4 (5), 2054-2061.

10. Wang, Y.; Huang, N. Y.; Zhang, X. W.; He, H.; Huang, R. K.; Ye, Z. M.; Li, Y.; Zhou, D. D.; Liao, P. Q.; Chen, X. M.; Zhang, J. P., Selective Aerobic Oxidation of a Metal-Organic Framework Boosts Thermodynamic and Kinetic Propylene/Propane Selectivity. Angew. Chem. Int. Ed. Engl. 2019, 58, 1-6.

11. Bae, Y.-S.; Lee, C. Y.; Kim, K. C.; Farha, O. K.; Nickias, P.; Hupp, J. T.; Nguyen, S. T.; Snurr, R. Q., High Propene/Propane Selectivity in Isostructural Metal-Organic Frameworks with High Densities of Open Metal Sites. Angewandte Chemie International Edition. 2012, 51 (8), 1857-1860.

12. Kim, S. Y.; Yoon, T. U.; Kang, J. H.; Kim, A. R.; Kim, T. H.; Kim, S. I.; Park, W.; Kim, K. C.; Bae, Y. S., Observation of Olefin/Paraffin Selectivity in Azo 
Compound and Its Application into a Metal-Organic Framework. ACS Appl. Mater. Interfaces. 2018, 10 (32).

13. Campo, M. C.; Ribeiro, A. M.; Ferreira, A.; Santos, J. C.; Lutz, C.; Loureiro, J. M.; Rodrigues, A. E., New 13X zeolite for propylene/propane separation by vacuum swing adsorption. Sep. Purif. Technol. 2013, 103, 60-70.

14. Plaza, M. G.; Ferreira, A. F. P.; Santos, J. C.; Ribeiro, A. M.; Müller, U.; Trukhan, N.; Loureiro, J. M.; Rodrigues, A. E., Propane/propylene separation by adsorption using shaped copper trimesate MOF. Microporous Mesoporous Mater. 2012, 157, $101-111$

15. Wang, X.; Krishna, R.; Li, L.; Wang, B.; He, T.; Zhang, Y.-Z.; Li, J.-R.; Li, J., Guest-dependent pressure induced gate-opening effect enables effective separation of propene and propane in a flexible MOF. Chem. Eng. J. 2018, 346, 489-496.

16. Lee, C. Y.; Bae, Y. S.; Jeong, N. C.; Farha, O. K.; Sarjeant, A. A.; Stern, C. L.; Nickias, P.; Snurr, R. Q.; Hupp, J. T.; Nguyen, S. T., Kinetic separation of propene and propane in metal-organic frameworks: controlling diffusion rates in plate-shaped crystals via tuning of pore apertures and crystallite aspect ratios. J. Am. Chem. Soc. 2011, 133 (14), 5228-5231.

17. Peng, J.; Wang, H.; Olson, D. H.; Li, Z.; Li, J., Efficient kinetic separation of propene and propane using two microporous metal organic frameworks. Chem. Commun. (Camb.) 2017, 53 (67). 
18. Li, L. B.; Lin, R. B.; Wang, X. Q.; Zhou, W.; Jia, L. T.; Li, J. P.; Chen, B. L., Kinetic separation of propylene over propane in a microporous metal-organic framework. Chem. Eng. J. 2018, 354, 977-982.

19. Wu, H.; Yuan, Y.; Chen, Y.; Xu, F.; Lv, D.; Wu, Y.; Li, Z.; Xia, Q., Efficient adsorptive separation of propene over propane through a pillar - layer cobalt - based metal-organic framework. AIChE J. 2019, 16858. 